\title{
ORIGINAL
}

\section{Endocarditis infecciosa en la Unidad de Medicina Intensiva}

\author{
S. Miranda-Montero ${ }^{\mathrm{a}, *}$, M. Rodríguez-Esteban ${ }^{\mathrm{b}}$, L. Álvarez-Acosta ${ }^{\mathrm{b}}$, \\ S. Lubillo-Montenegro ${ }^{a}, H$. Pérez-Hernández ${ }^{b}$ y R. Llorens-León ${ }^{c}$ \\ a Servicio de Medicina Intensiva, Hospital Universitario Ntra. Sra. de Candelaria, Santa Cruz de Tenerife, España
b Servicio de Cardiología, Hospital Universitario Ntra. Sra. de Candelaria, Santa Cruz de Tenerife, España
c Servicio de Cirugía Cardiaca, Hospiten Rambla, Santa Cruz de Tenerife, España
}

Recibido el 13 de noviembre de 2011; aceptado el 3 de febrero de 2012

Disponible en Internet el 9 de mayo de 2012

\section{PALABRAS CLAVE \\ Endocarditis; \\ Enfermo crítico; \\ Unidad de Cuidados \\ Intensivos}

\begin{abstract}
Resumen
Objetivo: Conocer las características, evolución y pronóstico de los pacientes con endocarditis infecciosa que requieren tratamiento en la Unidad de Medicina Intensiva.

Diseño: Estudio observacional de cohortes prospectivo en pacientes ingresados por endocarditis infecciosa.

Ámbito: Hospital Universitario Nuestra Señora de Candelaria, centro con 824 camas y población asignada de 493.145 personas.

Pacientes: Todos los pacientes diagnosticados de endocarditis siguiendo los criterios de Duke entre el 1 de enero de 2005 y el 31 de julio de 2011.

Variables de interés: Variables demográficas, clínicas, scores de gravedad, hallazgos microbiológicos y ecocardiográficos, mortalidad intrahospitalaria y complicaciones.

Resultados: De 102 pacientes diagnosticados de endocarditis, 38 (37\%) ingresaron en Medicina Intensiva. Comparándolos con los que no lo hicieron, sufrieron con más frecuencia afectación mitral (OR= 7,13; IC del 95\%, 2,12-24; $p=0,002)$ y embolia cerebral (OR= 3,89; IC del 95\%, 1,06$14,3 ; p=0,041)$. La mortalidad fue mayor $(42,1$ vs $18,8 \%, p=0,011)$, así como la proporción de cirugías urgentes $(45,8$ vs $5,9 \%, p<0,001)$. Resultaron predictores de mortalidad la infección por Estafilococo aureus (OR=3,49; IC 95\%: 1,02-11,93; $p=0,046$ ), la insuficiencia cardiaca (OR=4,18; IC 95\%: 1,17-14,94; $p=0,028)$, el embolismo cerebral (OR= 8,45; IC 95\%: 1,89-37,74; $p=0,005)$ y la puntuación en el score SAPS II al ingreso (OR=1,09; IC 95\% 1,04-1,15; $p<0,001)$. Conclusiones: Una elevada proporción de pacientes con endocarditis requieren ingreso en la Unidad de Medicina Intensiva, presentando un pronóstico mucho más desfavorable. La infección por $E$. aureus, la insuficiencia cardiaca, el embolismo cerebral y la puntuación SAPS II resultan predictores de mortalidad intrahospitalaria.

๑ 2011 Elsevier España, S.L. y SEMICYUC. Todos los derechos reservados.
\end{abstract}

\footnotetext{
* Autor para correspondencia.

Correo electrónico: simirmon@hotmail.com (S. Miranda-Montero).
} 


\section{KEYWORDS}

Endocarditis;

Critically ill;

Intensive Care Unit
Infectious endocarditis in the intensive care unit

\begin{abstract}
Objective: To study the characteristics, evolution and prognosis of patients with infectious endocarditis requiring treatment in the Intensive Care Unit.

Design: A prospective, observational cohort study of patients admitted due to infectious endocarditis.

Setting: Nuestra Señora de Candelaria University Hospital, a third - level center with a recruitment population of 493,145.

Patients: All patients consecutively diagnosed with infectious endocarditis in our center according to the Duke criteria, between 1 January 2005 and 31 July 2011.

Study variables: Demographic data, clinical severity scores, microbiological and echocardiographic data, hospital mortality and complications.

Results: Out of 102 patients diagnosed with endocarditis, 38 (37\%) were admitted to Intensive Care. Compared with those patients not admitted to the ICU, these subjects suffered more frequent mitral valve alterations $(\mathrm{OR}=7.13 ; 95 \% \mathrm{Cl}: 2.12-24 ; \mathrm{p}=0.002)$ and cerebral embolism $(\mathrm{OR}=3.89 ; 95 \% \mathrm{Cl}: 1.06-14.3 ; \mathrm{p}=0.041)$. In turn, mortality was greater $(42.1 \%$ vs $18.8 \%, \mathrm{p}=0.011)$, as was the proportion of emergency surgeries $(45.8 \%$ vs $5.9 \%, p<0.001)$. The identified mortality predictors were Staphylococcus aureus infection $(O R=3.49 ; 95 \% \mathrm{Cl} 1.02-11.93 ; \mathrm{p}=0.046)$, heart failure $(O R=4.18 ; 95 \% \mathrm{Cl}: 1.17-14.94 ; \mathrm{p}=0.028)$, cerebral embolism $(\mathrm{OR}=8.45 ; 95 \% \mathrm{Cl}: 1.89$ 37.74; $p=0.005)$ and the SAPS II upon admission (OR=1.09; 95\% Cl: 1.04-1.15; $p<0.001)$.

Conclusions: A large proportion of patients with endocarditis require admission to the Intensive Care Unit, presenting a much poorer prognosis. Staphylococcus aureus infection, heart failure, cerebral embolism and SAPS II scores are independent predictors of hospital mortality.

(c) 2011 Elsevier España, S.L. and SEMICYUC. All rights reserved.
\end{abstract}

\section{Introducción}

La endocarditis infecciosa (EI) es una enfermedad poco frecuente, con un manejo especialmente difícil y que requiere habitualmente la colaboración estrecha de intensivistas, especialistas en enfermedades infecciosas, cardiólogos y cirujanos cardiacos para intentar mejorar los resultados del tratamiento ${ }^{1}$. Es evidente que se han producido cambios importantes en los patrones epidemiológicos y microbiológicos, especialmente en los hospitales de tercer nivel de países desarrollados, con una mayor proporción de pacientes de edad avanzada y pluripatológicos que demandan tratamiento, con infección por gérmenes agresivos y presentación aguda $^{2-4}$. Si bien la necesidad de un manejo multidisciplinario es algo que hoy en día prácticamente nadie duda, se hace poca mención a los pacientes que precisan ingreso en la Unidad de Medicina Intensiva (UMI), a pesar de la alta frecuencia de complicaciones graves que se presentan en el transcurso de la infección ${ }^{5,6}$; los enfermos pueden ingresar en la UMI desde urgencias o desde otros servicios como Cardiología o Medicina Interna cuando el estado de gravedad requiere la ayuda de estas unidades especializadas. La mortalidad hospitalaria de los pacientes con sepsis grave o shock séptico se ha situado entre el 20 y $50 \%$, dato probablemente infraestimado y ensombrecido por una derivación tardía a la UMI, aún cuando se han establecido claros criterios de ingreso en las unidades de cuidados intensivos para pacientes con esta situación ${ }^{7}$. En el caso de la endocarditis, una serie amplia y reciente analizada en nuestro país muestra una mortalidad intrahospitalaria del $29,5 \%^{8}$.

El objetivo de nuestro trabajo es analizar las características diferenciales de los pacientes con $\mathrm{El}$ que requieren ingreso en la UMI, así como la evolución y el pronóstico intrahospitalario de los enfermos atendiendo a criterios y scores objetivos de gravedad, identificando las variables que se asocian con el ingreso en la Unidad y con la muerte de los pacientes.

\section{Metodología}

Realizamos un estudio observacional de cohortes prospectivo en los pacientes ingresados de forma consecutiva por endocarditis infecciosa o que desarrollaron esta enfermedad durante su estancia en el Hospital Universitario Nuestra Señora de Candelaria, centro de tercer nivel con 824 camas funcionantes, que cubre la mayor parte de la población de la Isla de Tenerife (493.145 personas) y que representa el hospital de referencia de las Islas de La Gomera y El Hierro.

\section{Pacientes}

Enfermos diagnosticados de $\mathrm{El}$ en nuestro centro entre el 1 de enero de 2005 y el 31 de julio de 2011. Todos cumplían los criterios de endocarditis definitiva o probable de acuerdo con la clasificación de Duke ${ }^{9}$. En todos se elaboró una historia médica que incluía sus antecedentes patológicos, estudio microbiológico, variables ecocardiográficas, desarrollo de complicaciones de la enfermedad y la valoración del estado de gravedad siguiendo la puntuación de las escalas APACHE II (Acute Phisiology And Chronic Health Evaluation II) ${ }^{10}$, SAPS II (Simplified Acute Physiology Score II) ${ }^{11}$ y SOFA (Sepsis-related Organ Failure Assessment) ${ }^{12}$. 


\section{Definiciones}

Consideramos como variables dependientes el ingreso en UMI (cualquier estancia en la Unidad, basados en la necesidad de tratar al menos el fallo de un órgano y excluyendo la permanencia habitual tras la cirugía cardiaca) y la mortalidad intrahospitalaria, incluyendo la acontecida antes de abandonar el hospital fuera el paciente intervenido quirúrgicamente o no.

La El aguda se definió como aquel episodio de menos de 2 semanas de evolución desde la aparición de los primeros signos y síntomas. Se consideró el desarrollo de insuficiencia renal aguda siguiendo los criterios RIFLE para daño o insuficiencia renal. Solo consideramos las clases III y IV de la New York Heart Association (NYHA) cuando hablamos del desarrollo de insuficiencia cardiaca. El embolismo cerebral o periférico debía constatarse mediante pruebas de imagen (TAC, RM o ecografía) para considerarlo como tal. La cirugía urgente se refiere a aquella que no se demoró más de 24 horas tras el establecimiento de la indicación.

\section{Exámenes complementarios}

En todos los pacientes se extrajeron al menos 3 hemocultivos, y se realizó serología de Brucella, Legionella, Mycoplasma y Coxiella burnetii en caso de hemocultivos negativos $\mathrm{y} / \mathrm{o}$ bajo criterio del médico responsable.

En el estudio ecocardiográfico transtorácico y transesofágico, se consideró la localización de las vegetaciones en caso de que estuvieran presentes, la presencia de una nueva insuficiencia valvular igual o mayor de 2/4, la aparición de una nueva dehiscencia protésica y el desarrollo de complicaciones perianulares (absceso, pseudoaneurisma o fístula).

Salvo que el estado de gravedad del paciente no lo permitiera, se realizó una ecografía o un escáner de abdomen y un escáner de cráneo en busca de fenómenos embólicos.

\section{Análisis estadístico}

Los datos se presentan como porcentaje o como media \pm desviación estándar 0 mediana (rango intercuartílico), según proceda. Se determinó la normalidad en la distribución de las variables continuas mediante el test de Kolmogorov-Smirnoff. Las variables categóricas se expresan como frecuencia absoluta (porcentaje) y se compararon con el test de la Chi-cuadrado o el test exacto de Fisher en caso de ser necesario. Para las variables continuas se utilizó el test de la $t$ de Student para datos no apareados o el de la $U$ de Mann Whitney cuando era apropiado. Para las diferencias evolutivas en los scores de gravedad se empleó el test de Wilcoxon. Se realizó un test de regresión logística con el método de selección hacia delante (razón de verosimilitud) con las variables que mostraron un valor de $\mathrm{p}$ menor de 0,05 para identificar los factores de riesgo para ingreso en UMI y de mortalidad intrahospitalaria. Se efectuó la prueba de bondad del ajuste mediante el test de Hosmer-Lemeshow. El análisis se desarrolló con el paquete estadístico SPSS (versión 16.0).

\section{Resultados}

\section{Características diferenciales de los pacientes ingresados en UMI}

En el periodo estudiado fueron diagnosticados de El en nuestro centro 102 pacientes. En 38 de ellos se consideró necesario el ingreso en la UMI. De estos, 25 (65,8\%) procedían directamente de urgencias, $7(18,4 \%)$ del servicio de Cardiología, $3(7,9 \%)$ de Medicina Interna y los 3 restantes de otros servicios.

Entre las causas que motivaron la terapia intensiva predominan la sepsis grave o el shock séptico (14 pacientes, $36,8 \%$ ) y la insuficiencia cardiaca grave o el shock cardiogénico (14 casos, 36,8\%). En 3 enfermos (7,9\%) la causa de ingreso fue el deterioro del estado neurológico. En los restantes ( 7 pacientes, $18,4 \%$ ) los motivos fueron variados, aunque relacionados con la gravedad del paciente, e incluyeron parada cardiorrespiratoria reanimada y neumonía. Diecinueve $(50 \%)$ de los enfermos requirieron ventilación mecánica.

Las diferencias demográficas y clínicas más relevantes entre los pacientes que ingresaron en la UMI y los que no lo hicieron se presentan en la tabla 1. Los pacientes con estancia en la Unidad de Intensivos presentan con más frecuencia una endocarditis aguda, afectación mitral y es más habitual que la enfermedad se vea complicada con insuficiencia cardiaca y con fenómenos embólicos. La puntuación en los modelos predictivos de mortalidad (SAPS II, APACHE II, SOFA) al ingreso hospitalario también fue mayor en caso de ingreso en UMI.

Si bien no hay diferencias en la indicación global de cirugía, se enviaron de forma urgente a quirófano desde intensivos a 11 enfermos (45,8\% de los intervenidos) frente a $2(5,9 \%)$ desde otros servicios médicos $(p<0,001)$. Las causas que motivaron la cirugía fueron principalmente la insuficiencia cardiaca $(36,2 \%)$, la destrucción del aparato valvular con insuficiencia severa $(27,6 \%)$ y la fiebre persistente $(15,5 \%)$. Otras causas incluyeron la afectación anular o la presencia de vegetaciones de gran tamaño.

En cuanto al agente etiológico, en los pacientes que ingresaron en la UMI predomina claramente el Estafilococo aureus, siendo significativamente más frecuente que en los no ingresados en la Unidad $(39,5$ vs $20,3 \%, p=0,036)$. Le siguen el Estreptococo $(18,4 \%)$, el Enterococo $(10,5 \%)$ y otros agentes $(7,9 \%)$. Los Estafilococos coagulasa negativo son más raros en comparación con los pacientes no ingresados $(5,3$ vs $20,3 \%, p=0,038)$. Por último, los enfermos con hemocultivos negativos representan el $18,4 \%$ en la UMI.

En la tabla 2 se especifican las características ecocardiográficas. No existen diferencias significativas en la proporción de endocarditis protésicas, frecuencia de afectación anular ni de insuficiencia valvular moderada o severa. Sin embargo, en la UMI es mayor el porcentaje de enfermos con afectación mitral y no se registraron casos de endocarditis tricuspídea durante el periodo estudiado.

En el análisis multivariable se presentan como predictores de ingreso el embolismo cerebral (OR= 3,89; IC del $95 \%, 1,06-14,3 ; p=0,041)$, la puntuación SOFA al ingreso hospitalario $(O R=1,60 ; \mathrm{IC}$ del 95\%, 1,29-2,00; $p<0,001)$ y la afectación de la válvula mitral $(\mathrm{OR}=7,13$; IC del $95 \%$, 
Tabla 1 Características clínicas y complicaciones

\begin{tabular}{|c|c|c|c|c|}
\hline & Total $(n=102)$ & UMI $(n=38)$ & No UMI $(n=64)$ & $\mathrm{p}$ \\
\hline Edad & $60,9 \pm 15.4$ & $58,6 \pm 12,7$ & $62,4 \pm 16.7$ & 0,2 \\
\hline Hombres & $79(77,5)$ & $25(65,8)$ & $54(84,4)$ & 0,03 \\
\hline ADVP & $5(4,9)$ & $1(2,6)$ & $4(6,3)$ & 0,65 \\
\hline DM & $32(31,4)$ & $8(21,1)$ & $24(37,5)$ & 0,083 \\
\hline Hemodiálisis & $8(7,8)$ & $3(7,9)$ & $5(7,8)$ & 0,988 \\
\hline Presentación aguda & $51(50)$ & $25(65,8)$ & $26(40,6)$ & 0,014 \\
\hline ICC & $51(50)$ & $26(68,4)$ & $25(39,1)$ & 0,004 \\
\hline Embolia cerebral & $18(17,6)$ & $10(26,3)$ & $8(12,5)$ & 0,077 \\
\hline Embolia periférica & $33(32,4)$ & $17(44,7)$ & $16(25)$ & 0,039 \\
\hline IRA & $43(42,2)$ & $21(55,3)$ & $22(34,4)$ & 0,039 \\
\hline APACHE II inicial & $9(5-14)$ & $12,5(7-17,25)$ & $8(5-10,75)$ & 0,001 \\
\hline SOFA inicial & $1(0-4,25)$ & $3(1-7,25)$ & $0,5(0-2)$ & $<0,001$ \\
\hline SAPS II inicial & $24(18-32)$ & $30(22,75-42,5)$ & $22(18-27)$ & $<0,001$ \\
\hline Cirugía & $58(56,9)$ & $24(63,2)$ & $34(53,1)$ & 0,323 \\
\hline Urgente & $13(22,4)$ & $11(45,8)$ & $2(5,9)$ & $<0,001$ \\
\hline Exitus & $28(27,5)$ & $16(42,1)$ & $12(18,8)$ & 0,011 \\
\hline
\end{tabular}

Los datos se expresan como media \pm desviación estándar, mediana (rango intercuartílico) o n (\%). ADVP: adictos a drogas por vía parenteral; DM: diabetes mellitas; ICC: insuficiencia cardiaca; IRA: insuficiencia renal aguda; No UMI: pacientes que no ingresaron en dicha unidad; UMI: Pacientes ingresados en la Unidad de Medicina Intensiva.

2,12-24; $\mathrm{p}=0.002)$. El test de Hosmer - Lemeshow presentó un valor de $p=0,87$.

\section{Mortalidad intrahospitalaria}

La mortalidad intrahospitalaria alcanzó el $42,1 \%$ en UMI frente al $18,8 \%$ de los pacientes que no ingresaron en ella $(p=0,011)$. Como causa del exitus predominó el shock séptico $(51,8 \%)$, seguido de la insuficiencia cardiaca grave o shock cardiogénico $(25,9 \%)$ y las complicaciones neurológicas $(14,8 \%)$.

Los enfermos que fallecieron durante el ingreso presentaban en el análisis univariable una edad más avanzada, mayor prevalencia de diabetes mellitus, infección por $E$. aureus con más frecuencia y se complicaron habitualmente con insuficiencia cardiaca, con embolia cerebral y con insuficiencia renal. Los valores de las escalas de gravedad al ingreso también eran significativamente mayores. A pesar de que el porcentaje de operados fue mayor en los supervivientes, no se alcanzaron diferencias significativas. La tabla 3 representa las diferencias principales entre los pacientes que sobrevivieron o no la fase hospitalaria.

En el análisis multivariable (tabla 4) se presentaron como predictores independientes de mortalidad la infección por E. aureus $(\mathrm{OR}=3,49$; IC del 95\%, 1,02-11,93; $\mathrm{p}=0,046)$, el desarrollo de insuficiencia cardiaca $(O R=4,18$; IC del $95 \%$, $1,17-14,94 ; p=0,028)$, el embolismo cerebral $(O R=8,45$; IC del $95 \%, 1,89-37,74 ; p=0,005)$ y la puntuación en la escala SAPS II (OR=1,09; IC del 95\%, 1,04-1,15; $p<0,001)$. El test de Hosmer-Lemeshow mostró un valor de $p=0,81$.

\section{Discusión}

Aunque se asume que el deterioro hemodinámico y el fallo de uno o más órganos puede necesitar el tratamiento y vigilancia intensivas, la proporción exacta de pacientes con endocarditis que requiere ingreso en la Unidad de Críticos no es bien conocida. Es más, el número de casos puede

Tabla 2 Características diferenciales ecocardiográficas

\begin{tabular}{|c|c|c|c|c|}
\hline & Total $(n=102)$ & UMI $(n=38)$ & No UMI $(n=64)$ & $\mathrm{p}$ \\
\hline \multicolumn{5}{|l|}{ Localización } \\
\hline Mitral & $24(23,5)$ & $15(39,5)$ & $9(14,1)$ & 0,007 \\
\hline Aórtica & $43(42,2)$ & $21(55,3)$ & $22(34,4)$ & 0,061 \\
\hline Tricúspide & $8(7,8)$ & $0(0)$ & $8(12,5)$ & 0,024 \\
\hline MCP/DAI & $11(10,8)$ & $2(5,3)$ & $9(14,1)$ & 0,204 \\
\hline Otras & $3(2,9)$ & $1(2,6)$ & $2(3,1)$ & 1 \\
\hline Protésica & $21(20,6)$ & $5(13,2)$ & $16(25)$ & 0.207 \\
\hline Afectación anular & $20(19,6)$ & $8(21,1)$ & $12(18,8)$ & 0,777 \\
\hline Insuf.valvular $\geq 2 / 4$ & $60(58,8)$ & $25(65,8)$ & $35(54,7)$ & 0,271 \\
\hline
\end{tabular}

Los datos se expresan como n (\%). Insuf. valvular: insuficiencia valvular; MCP/DAl: sobre marcapasos o desfibrilador implantable; No UMI: pacientes que no ingresaron en dicha unidad; UMI: pacientes ingresados en la Unidad de Medicina Intensiva. 
Tabla 3 Influencia de distintas variables en la mortalidad intrahospitalaria. Análisis univariado

\begin{tabular}{|c|c|c|c|}
\hline & Exitus $(n=28)$ & Supervivientes $(n=74)$ & $\mathrm{p}$ \\
\hline Edad & $66,7 \pm 12,0$ & $58,8 \pm 16,0$ & 0,019 \\
\hline Hombres & $19(67,9)$ & $60(81,1)$ & 0,154 \\
\hline$A D V P$ & $1(3,6)$ & $4(5,4)$ & 1 \\
\hline$D M$ & $13(46,4)$ & $19(25,7)$ & 0,044 \\
\hline Hemodiálisis & $3(10,7)$ & $5(6,8)$ & 0,507 \\
\hline Presentación aguda & $16(57,1)$ & $35(47,3)$ & 0,375 \\
\hline \multicolumn{4}{|l|}{ Localización } \\
\hline Mitral & $7(25)$ & $17(23)$ & 0,8 \\
\hline Aórtica & $12(42,9)$ & $31(41,9)$ & 1 \\
\hline Tricúspide & $2(7,1)$ & $6(8,1)$ & 1 \\
\hline MCP/DAI & $2(7,1)$ & $9(12,2)$ & 0,723 \\
\hline Otras & $1(3,6)$ & $2(2,7)$ & 1 \\
\hline Protésica & $4(14,3)$ & $17(23)$ & 0,333 \\
\hline Afectación anular & $6(21,4)$ & $14(18,9)$ & 0,776 \\
\hline \multicolumn{4}{|l|}{ Germen } \\
\hline S. aureus & $13(46,4)$ & $15(20,3)$ & 0,013 \\
\hline S. coagulasa neg. & $1(3,6)$ & $14(18,9)$ & 0,062 \\
\hline Streptococcus & $4(14,3)$ & $23(31,1)$ & 0,130 \\
\hline Enterococcus & $4(14,3)$ & $12(16,2)$ & 1 \\
\hline Hemoc. negativo & $4(14,3)$ & $8(10,8)$ & 0,732 \\
\hline Otros & $1(3,6)$ & $3(4,2)$ & 1 \\
\hline$I C C$ & $22(78,6)$ & $29(39,2)$ & $<0,001$ \\
\hline Insuf.valvular $\geq 2 / 4$ & $18(64,3)$ & $42(56,8)$ & 0,491 \\
\hline Embolia cerebral & $9(32,1)$ & $9(12,2)$ & 0,018 \\
\hline Embolia periférica & $10(35,7)$ & $23(31,1)$ & 0,655 \\
\hline IRA & $18(64,3)$ & $25(33,8)$ & 0,005 \\
\hline APACHE II inicial & $13(7,25-21)$ & $8(5-11)$ & $<0,001$ \\
\hline SOFA inicial & $4,5(1-7,75)$ & $1(0-2,25)$ & $<0,001$ \\
\hline SAPS II inicial & $36(24-49)$ & $22(16-27)$ & $<0,001$ \\
\hline Cirugía & $12(42,9)$ & $46(66,2)$ & 0,079 \\
\hline Creatinina & $1,77(1-2,67)$ & $1(0,85-1,41)$ & 0,001 \\
\hline Leucocitos & $11.750(8.075-24.087)$ & $11.300(8.400-16.000)$ & 0,345 \\
\hline
\end{tabular}

estar infradiagnosticado y en ese sentido la ampliación de realización de ecocardiogramas transtorácicos y sobre todo transesofágicos en pacientes con sepsis podría resultar de gran utilidad ${ }^{13}$. En nuestro hospital, más de una tercera parte de los pacientes con diagnóstico de endocarditis en un periodo mayor de 6 años fue atendido en la UMI por diferentes causas, fundamentalmente sepsis e insuficiencia cardiaca graves complicados o no con shock. La diferencia en los scores de gravedad entre los pacientes con y sin ingreso en la UMI y el aumento en los mismos desde la llegada del paciente al hospital muestran de forma objetiva el empeoramiento clínico y hemodinámico que presentaban los enfermos y que pueden reflejarse en la elevada morbimortalidad y frecuencia en la indicación de cirugía urgente.

En la serie de Karth et al. ${ }^{14}$ se analizaron 33 pacientes con ingreso en la Unidad de Cuidados Intensivos a causa de un curso complicado en un periodo de 4 años (1994-1999), con una edad, porcentaje de pacientes sometidos a cirugía y perfil microbiológico muy parecido al de nuestro centro y la mortalidad intrahospitalaria alcanzó el $54 \%$. Por otra parte, en la amplia serie retrospectiva de Mourvillier et al. ${ }^{15}$, realizada en un periodo similar (19932000) el porcentaje de exitus fue del 45\%, ligeramente superior al nuestro. En cualquier caso, existe una clara

Tabla 4 Predictores independientes de mortalidad intrahospitalaria

\begin{tabular}{lllr}
\hline & OR & IC del 95\% & \multicolumn{1}{c}{$\mathrm{P}$} \\
\hline S. aureus & 3,49 & $1,02-11,93$ & 0,046 \\
Insuficiencia cardiaca & 4,18 & $1,17-14,94$ & 0,028 \\
Embolismo cerebral & 8,45 & $1,89-37,74$ & 0,005 \\
SAPS II & 1,09 & $1,04-1,15$ & $<0,001$ \\
\hline
\end{tabular}

IC del 95\%: intervalo de confianza del 95\%; OR: odds ratio; SAPS II: Simplified Acute Physiology Score II; Test de Hosmer-Lemeshow: $p=0,87$. 
diferencia con la mortalidad de los pacientes no ingresados en UMI, que en nuestra serie no alcanza el 20\%. Esta diferencia se puede explicar por la mayor gravedad objetiva y por un perfil microbiológico más adverso. Cuando se analizan los predictores de mortalidad en la endocarditis pueden encontrarse grandes diferencias entre los diversos trabajos publicados, probablemente debido a lo heterogéneo de las muestras y al curso imprevisible que en muchos casos sigue la enfermedad. En un amplio estudio multinacional reciente se presentaron como predictores de mortalidad intrahospitalaria la infección por $E$. aureus, la afectación mitral o protésica, las complicaciones perivalvulares y el edema pulmonar ${ }^{2}$. En otra cohorte multicéntrica de centros españoles ${ }^{8}$ con un periodo de estudio que abarca desde 1984 hasta 2006, y con una mortalidad global del $29,5 \%$, los factores independientes fueron el score de morbilidad de Charlson, las manifestaciones neurológicas, la insuficiencia renal aguda, el shock séptico y, de nuevo, la afectación protésica y perivalvular, la insuficiencia cardiaca y la infección por Estafilococo. En nuestro trabajo se repiten como predictores la infección por este germen, el desarrollo de fallo cardiaco y las complicaciones neurológicas. Sin embargo, los scores que se utilizan como predictores de mortalidad hospitalaria, de uso común en las UMI, han sido empleados con poca frecuencia en la literatura para la valoración del pronóstico de la El. La puntuación de la escala SAPS II al ingreso hospitalario se muestra como un predictor independiente de mortalidad en la presente serie, como lo fue la escala APACHE II en la serie de Chu et al. ${ }^{16}$. Cabría pensar aquí que la evaluación de estos scores al ingreso del paciente con El podría hacernos prever el nivel de cuidados que podría requerir, sin bien habría que diseñar un análisis más amplio al efecto.

El porcentaje de supervivientes es mayor en el grupo de pacientes sometidos a intervención, aunque la diferencia no alcanza significación estadística. Dado el valor de $\mathrm{p}$ se decidió incluirlo en un modelo multivariable sin que tampoco se presentara como predictor. Quizás la ausencia de un beneficio uniforme en los pacientes que van a quirófano pudiera desaparecer con una selección adecuada de los mismos ${ }^{17-18}$. A pesar de que la cirugía juega un papel primordial en el tratamiento de la El, la decisión de utilizarla y el momento en que se realiza se ve influenciada por múltiples factores que incluyen la comorbilidad del enfermo, la edad, el tratamiento antibiótico previo o la disponibilidad de un equipo quirúrgico con experiencia ${ }^{19}$. Las guías de endocarditis de la Sociedad Europea de Cardiología recogen como indicaciones de cirugía la insuficiencia cardiaca severa, los signos de mala tolerancia hemodinámica, la fiebre persistente, los signos de infección local no controlada como los abscesos, la regurgitación mitral o aórtica severa y la presencia de vegetaciones grandes tras uno o más episodios embólicos a pesar de tratamiento médico adecuado ${ }^{20}$. Creemos que las indicaciones establecidas en nuestro centro se ajustan a estas recomendaciones.

\section{Conclusiones}

En nuestro medio, la UMI colabora en el manejo del enfermo con endocarditis infecciosa en una alta proporción de casos. Estos presentan un mayor número de complicaciones y una gravedad objetivamente mayor que se traduce en una mortalidad elevada y una alta tasa de cirugía urgente. Son predictores independientes de mortalidad intrahospitalaria la infección por $E$. aureus, el desarrollo de insuficiencia cardiaca, el embolismo cerebral y la puntuación en el score SAPS II al ingreso.

\section{Conflicto de intereses}

Los autores declaran no tener ningún conflicto de intereses.

\section{Bibliografía}

1. Tornos P. Endocarditis infecciosa: una enfermedad grave e infrecuente que requiere ser tratada en hospitales con experiencia. Rev Esp Cardiol. 2005;58:1145-7.

2. Murdoch DR, Corey GR, Hoen B, Miró JM, Fowler VG, Bayer AS, et al. Clinical presentation, etiology, and outcome of infective endocarditis in the 21st century. The International Collaboration on Endocarditis-Prospective Cohort Study. Arch Intern Med. 2009;169:463-73.

3. Tleyjeh IM, Abdel-Latif A, Rahbi H, Scott CG, Bailey KR, Steckelberg JM, et al. A systematic review of population-based studies of infective endocarditis. CHEST. 2007;13:1025-35.

4. Cabell CH, Jollis JG, Peterson GE, Corey GR, Anderson DJ, Sexton DJ, et al. Changing patient characteristics and the effect on mortality in endocarditis. Arch Intern Med. 2002;162:90-4.

5. Maroto F, Gallego S, Pérez C, Colon C. Endocarditis infecciosa en enfermedad por mordedura de rata. Med Intensiva. 2011;35:317-8.

6. Alemparte-Pardavilla E, González-Chana B, Cortés-Cañones JR. Endocarditis fúngica sobre válvulas nativas. Med Intensiva. 2010;34:491-2.

7. Olaechea PM, Álvarez-Lerma F, Sánchez M, Torres A, Palomar M, Fernández $P$, et al. Evaluación del estado de gravedad de pacientes con infecciones graves. Criterios de ingreso en unidades de cuidados intensivos. Enferm Infecc Microbiol Clin. 2009;27:342-52.

8. Galvez-Acebal J, Rodríguez-Baño J, Martínez-Marcos J, Reguera JM, Plata A, Ruiz J, et al. Prognostic factors in leftsided endocarditis: results from the andalusian multicenter cohort. BMC Infectious Diseases. 2010;10:17.

9. Durack DT, Lukes AS, Bright DK. New criteria for diagnosis of infective endocarditis: utilization of specific echocardiographic findings. Duke Endocarditis Service. Am J Med. 1994;96:200-9.

10. Knaus WA, Draper EA, Wagner DP, Zimmerman JE. APACHE II: a severity of disease classification system. Critical Care Medicine. 1985;13:818-29.

11. Le Gall JR, Lemeshow S, Saulnier F. A New Simplified Acute Physiology Score (SAPS II) Based on a European/North American Multicenter Study. JAMA. 1993;270:2957-63.

12. Vincent JL, Moreno R, Takala J, Willatts S, De Mendonça A, Bruining $\mathrm{H}$, et al. The SOFA (Sepsis-related Organ Failure Assessment) score to describe organ dysfunction/failure. On behalf of the Working Group on Sepsis-Related Problems of the European Society of Intensive Care Medicine. Intensive Care Med. 1996;22:707-10.

13. Garcia-Vicente E, Campos-Nogué A, Gobernando-Serrano MM. Ecocardiografía en la Unidad de Cuidados Intensivos. Med Intensiva. 2008;32:236-47.

14. Karth GD, Koreny $M$, Binder $T$, Zauner $C$, Valentin $A$, Honninger R, et al. Complicated infective endocarditis necessitating ICU admission: clinical course and prognosis. Critical Care. 2002;6:149-54.

15. Mourvillier B, Trouillet JL, Timsin JF, Baudot J, Chastre J, Régnier $\mathrm{B}$, et al. Infective endocarditis in the intensive care 
unit: clinical spectrum and prognostic factors in 228 consecutive patients. Intensive Care Med. 2004;30:2046-52.

16. Chu VH, Cabell CH, Benjamin DK, Kuniholm EF, Fowler VG, Engemann J, et al. Early predictors of in-hospital death in infective endocarditis. Circulation. 2004;109:1745-9.

17. Cabell CH, Abrutyn E, Fowler VG, Hoen B, Miro JM, Corey GR. Use of surgery in patients with native valve infective endocarditis: results from the International Collaboration on Endocarditis Merged Database. Am Heart J. 2005;150:1092-8.
18. Delahaye F. Is early surgery beneficial in infective endocarditis? A systematic review. Arch Cardiovasc Dis. 2011;104: 35-44.

19. Prendergast BD, Tornos $P$. Surgery for infectiveEndocarditis. Who and When? Circulation. 2010;121:1141.

20. Habib G, Hoen B, Tornos P, Thuny F, Prendergast b Vilacosta I, et al. Guidelines for the prevention, diagnosis, and treatment of infective endocarditis. Eur Heart J. 2009;30: 2369-413. 